\title{
Boundary Relations and Generalized Resolvents of Symmetric Operators in Krein Spaces
}

\author{
Jussi Behrndt and Hans-Christian Kreusler
}

\begin{abstract}
The classical Krein-Naimark formula establishes a one-to-one correspondence between the generalized resolvents of a closed symmetric operator in a Hilbert space and the class of Nevanlinna families in a parameter space. Recently it was shown by V.A. Derkach, S. Hassi, M.M. Malamud and H.S.V. de Snoo that these parameter families can be interpreted as so-called Weyl families of boundary relations, and a new proof of the Krein-Naimark formula in the Hilbert space setting was given with the help of a coupling method. The main objective of this paper is to generalize the notion of boundary relations and their Weyl families to the Krein space case and to proof some variants of the Krein-Naimark formula in an indefinite setting.
\end{abstract}

Mathematics Subject Classification (2000). Primary: 47B50, 47A20, 47B25; Secondary: 46C20, 47A06.

Keywords. Symmetric operator, self-adjoint extension, Krein-Naimark formula, generalized resolvent, boundary relation, boundary triplet, (locally) definitizable operator, Krein space.

\section{Introduction}

Let $A$ be a closed symmetric operator with equal (possibly infinite) deficiency indices in a Hilbert space $\mathcal{K}$ and let $\left\{\mathcal{G}, \Gamma_{0}, \Gamma_{1}\right\}$ be a boundary triplet for the adjoint operator $A^{*}$. Let $A_{0}$ be the self-adjoint extension of $A$ in $\mathcal{K}$ corresponding to the boundary mapping $\Gamma_{0}, A_{0}=A^{*}\left\lceil\operatorname{ker} \Gamma_{0}\right.$, and denote the $\gamma$-field and Weyl function corresponding to $\left\{\mathcal{G}, \Gamma_{0}, \Gamma_{1}\right\}$ by $\gamma$ and $M$, respectively. Here the Weyl function $M$ is an $\mathcal{L}(\mathcal{G})$-valued Nevanlinna function with the additional property $0 \in \rho(\operatorname{Im} M(\lambda)), \lambda \in \mathbb{C} \backslash \mathbb{R}$. It is well known that in this case the Krein-Naimark formula

$$
\left.P_{\mathcal{K}}(\widetilde{A}-\lambda)^{-1}\right|_{\mathcal{K}}=\left(A_{0}-\lambda\right)^{-1}-\gamma(\lambda)(M(\lambda)+\tau(\lambda))^{-1} \gamma(\bar{\lambda})^{*}
$$


establishes a bijective correspondence between the class of Nevanlinna families $\tau$ in the parameter space $\mathcal{G}$ and the compressed resolvents of self-adjoint extensions $\widetilde{A}$ of $A$ in $\mathcal{K} \times \mathcal{H}$, where $\mathcal{H}$ is a Hilbert space. This description of the generalized resolvents of a symmetric operator was originally given by M.G. Krein and M.A. Naimark in $[25,26,28]$ for the case that $A$ is densely defined and has finite deficiency indices; see $[8,10,11,12,13,24,27]$ for our more general situation. Various generalizations of the Krein-Naimark formula in an indefinite setting have been proved in the last decades. E.g. the case that $A$ is a symmetric operator in a Pontryagin space $\mathcal{K}$ and $\mathcal{H}$ is a Hilbert space was investigated by M.G. Krein and H. Langer in [21]. Later V.A. Derkach considered both $\mathcal{K}$ and $\mathcal{H}$ to be Pontryagin or even Krein spaces, cf. [7]. Under additional assumptions other variants of (1.1) were proved in $[3,4,5,6,7,22]$.

Recently a very interesting new proof of the Krein-Naimark formula in the Hilbert space case was given in $[10,11]$ by V.A. Derkach, S. Hassi, M.M. Malamud and H.S.V. de Snoo with the help of a coupling method which allows to interpret the parameter family $\tau$ as a so-called Weyl family associated to a boundary relation of a symmetric relation in the Hilbert space $\mathcal{H}$. The concept of boundary relations is a generalization of the notion of boundary triplets which has the essential advantage that every Nevanlinna family can be realized as the Weyl family associated to a boundary relation, see [9].

The basic aim of this paper is to introduce the concept of boundary relations for symmetric relations in Krein spaces and to prove some variants of (1.1) in the Krein space case with a similar method as in $[8,10,11]$. Roughly speaking, if $A$ is a symmetric relation in a Krein space $\mathcal{K}$ which possesses a self-adjoint extension $A_{0}$ in $\mathcal{K}$ with a nonempty resolvent set, then we show in Theorem 3.1 that formula (1.1) gives a correspondence between compressed resolvents of selfadjoint extensions $\widetilde{A}$ in $\mathcal{K} \times \mathcal{H}$, where $\mathcal{H}$ is a Krein space, and the Weyl families $\tau$ corresponding to boundary relations of symmetric relations in $\mathcal{H}$. In contrast to the Hilbert space case where formula (1.1) makes sense for all $\lambda \in \mathbb{C} \backslash \mathbb{R}$ it is not immediately clear in our setting for which $\lambda \in \rho\left(A_{0}\right)$ the compressed resolvent of $\widetilde{A}$ and the inverse of $M+\tau$ are bounded operators on $\mathcal{K}$ and $\mathcal{G}$, respectively, cf. assertion (a) in Theorem 3.1 and Theorem 3.2. In the special situation that $A$ has finite defect, the fixed canonical extension $A_{0}$ locally (with the possible exception of a discrete set) has the same spectral properties as a self-adjoint operator in a Hilbert space and $\mathcal{H}$ is a Hilbert space we study the local spectral properties of $\widetilde{A}$ in Theorem 3.4, see also [3] for a similar situation.

The paper is organized as follows. Following the lines of [9] we introduce the concept of boundary relations and associated Weyl families for symmetric relations in Krein spaces in Section 2. The special case of boundary triplets and corresponding Weyl functions is briefly reviewed in Section 2.3. Section 3 contains our main results on Krein-Naimark type formulas in the Krein space setting discussed above. Finally, in Section 4 we show that certain classes of relation-valued functions can be realized as Weyl families corresponding to boundary relations of 
symmetric relations in Krein spaces. As a special case we obtain an alternative proof of the main result in [9], that is, each Nevanlinna family can be realized as the Weyl family of a boundary relation of a symmetric relation in a Hilbert space.

\section{Boundary relations of symmetric relations in Krein spaces}

The main objective of this section is to generalize the notion of boundary relations and associated Weyl families for symmetric relations in Hilbert spaces from [9] to symmetric relations in Krein spaces.

\subsection{Symmetric, self-adjoint, isometric and unitary relations in Krein spaces}

In the following let $\left(\mathcal{K},[\cdot, \cdot]_{\mathcal{K}}\right)$ and $\left(\mathcal{H},[\cdot, \cdot]_{\mathcal{H}}\right)$ be separable Krein spaces and let $J_{\mathcal{K}}$ and $J_{\mathcal{H}}$ be corresponding fundamental symmetries. The linear space of bounded linear operators defined on $\mathcal{K}$ with values in $\mathcal{H}$ is denoted by $\mathcal{L}(\mathcal{K}, \mathcal{H})$. If $\mathcal{K}=\mathcal{H}$ we simply write $\mathcal{L}(\mathcal{K})$. We study linear relations from $\mathcal{K}$ to $\mathcal{H}$, that is, linear subspaces of $\mathcal{K} \times \mathcal{H}$. The set of all closed linear relations from $\mathcal{K}$ to $\mathcal{H}$ is denoted by $\widetilde{\mathcal{C}}(\mathcal{K}, \mathcal{H})$. If $\mathcal{K}=\mathcal{H}$ we write $\widetilde{\mathcal{C}}(\mathcal{K})$. Linear operators from $\mathcal{K}$ into $\mathcal{H}$ are viewed as linear relations via their graphs. For the usual definitions of the linear operations with relations, the inverse etc., we refer to [14]. The domain (kernel, range, multivalued part) of a linear relation $S$ from $\mathcal{K}$ to $\mathcal{H}$ will be denoted by $\operatorname{dom} S(\operatorname{ker} S, \operatorname{ran} S$, mul $S$, resp.).

The resolvent set $\rho(S)$ of a closed linear relation $S \in \widetilde{\mathcal{C}}(\mathcal{K})$ is the set of all $\lambda \in \mathbb{C}$ such that $(S-\lambda)^{-1} \in \mathcal{L}(\mathcal{K})$, the spectrum $\sigma(S)$ of $S$ is the complement of $\rho(S)$ in $\mathbb{C}$. The extended spectrum $\widetilde{\sigma}(S)$ of $S$ is defined by $\widetilde{\sigma}(S)=\sigma(S)$ if $S \in \mathcal{L}(\mathcal{K})$ and $\widetilde{\sigma}(S)=\sigma(S) \cup\{\infty\}$ otherwise. The extended resolvent set $\widetilde{\rho}(S)$ of $S$ is defined by $\widetilde{\rho}(S)=\overline{\mathbb{C}} \backslash \widetilde{\sigma}(S)$. A point $\lambda \in \mathbb{C}$ is an eigenvalue of $S$ if $\operatorname{ker}(S-\lambda) \neq\{0\}$; we write $\lambda \in \sigma_{p}(S)$. We say that $\lambda \in \mathbb{C}$ belongs to the continuous spectrum $\sigma_{c}(S)$ (the residual spectrum $\left.\sigma_{r}(S)\right)$ of $S$ if $\operatorname{ker}(S-\lambda)=\{0\}, \operatorname{ran}(S-\lambda)$ is dense in $\mathcal{K}$ and $\operatorname{ran}(S-\lambda) \neq \mathcal{K}$ (resp. if $\operatorname{ker}(S-\lambda)=\{0\}$ and $\operatorname{ran}(S-\lambda)$ is not dense in $\mathcal{K})$. We set $\mathcal{N}_{\lambda, S}:=\operatorname{ker}(S-\lambda)$ and $\hat{\mathcal{N}}_{\lambda, S}:=\left\{\left(\begin{array}{c}g_{\lambda} \\ \lambda g_{\lambda}\end{array}\right) \mid g_{\lambda} \in \mathcal{N}_{\lambda, S}\right\}$.

If $U \subset \mathcal{K} \times \mathcal{H}$ is a linear relation from $\mathcal{K}$ to $\mathcal{H}$, then the adjoint relation $U^{+} \in \widetilde{\mathcal{C}}(\mathcal{H}, \mathcal{K})$ is defined by

$$
U^{+}:=\left\{\{\tilde{h}, \tilde{k}\} \in \mathcal{H} \times \mathcal{K} \mid[\tilde{h}, h]_{\mathcal{H}}=[\tilde{k}, k]_{\mathcal{K}} \text { for all }\{k, h\} \in U\right\} .
$$

The linear relation $U \subset \mathcal{K} \times \mathcal{H}$ is said to be isometric (unitary) if $U^{-1} \subset U^{+}$(resp. $\left.U^{-1}=U^{+}\right)$. If $A \subset \mathcal{K}^{2}$ is a linear relation in $\mathcal{K}$, then $A$ is said to be symmetric (self-adjoint) if $A \subset A^{+}$(resp. $\left.A=A^{+}\right)$. A unitary relation $U \in \widetilde{\mathcal{C}}(\mathcal{K}, \mathcal{H})$ satisfies

$$
\operatorname{ker} U=(\operatorname{dom} U)^{[\perp]_{\mathcal{K}}} \text { and } \operatorname{mul} U=(\operatorname{ran} U)^{[\perp]_{\mathcal{H}}}
$$

and $\operatorname{dom} U$ is closed if and only if ran $U$ is closed, see e.g. [9, Proposition 2.3].

A symmetric relation $A \in \widetilde{\mathcal{C}}(\mathcal{K})$ is said to be of defect $m \in \mathbb{N} \cup\{\infty\}$, if both deficiency indices

$$
n_{ \pm}\left(J_{\mathcal{K}} A\right)=\operatorname{dim} \operatorname{ker}\left(\left(J_{\mathcal{K}} A\right)^{*} \mp i\right)
$$


of the symmetric relation $J_{\mathcal{K}} A$ in the Hilbert space $\left(\mathcal{K},\left[J_{\mathcal{K}}, \cdot\right]_{\mathcal{K}}\right)$ are equal to $m$. Here ${ }^{*}$ denotes the adjoint with respect to the Hilbert scalar product $\left[J_{\mathcal{K}}, \cdot\right]_{\mathcal{K}}$. We note that the symmetric relation $A \in \widetilde{\mathcal{C}}(\mathcal{K})$ is of defect $m$ if and only if there exists a self-adjoint extension of $A$ in $\mathcal{K}$ and each self-adjoint extension $A^{\prime}$ of $A$ in $\mathcal{K}$ satisfies $\operatorname{dim}\left(A^{\prime} / A\right)=m$.

We define an indefinite inner product $\llbracket \cdot, \cdot \rrbracket_{\mathcal{K}^{2}}$ on $\mathcal{K}^{2}$ (and analogously $\llbracket \cdot, \cdot \rrbracket_{\mathcal{H}^{2}}$ on $\mathcal{H}^{2}$ ) by

$$
\llbracket \hat{f}, \hat{g} \rrbracket_{\mathcal{K}^{2}}=i\left(\left[f, g^{\prime}\right]_{\mathcal{K}}-\left[f^{\prime}, g\right]_{\mathcal{K}}\right), \quad \hat{f}=\left(\begin{array}{c}
f \\
f^{\prime}
\end{array}\right), \hat{g}=\left(\begin{array}{c}
g \\
g^{\prime}
\end{array}\right) \in \mathcal{K}^{2} .
$$

Then $\left(\mathcal{K}^{2}, \llbracket \cdot, \cdot \rrbracket_{\mathcal{K}^{2}}\right)$ is a Krein space and $\left(\begin{array}{cc}0 & -i J_{\mathcal{K}} \\ i J_{\mathcal{K}} & 0\end{array}\right) \in \mathcal{L}\left(\mathcal{K}^{2}\right)$ is a corresponding fundamental symmetry. Observe that also in the special case when $(\mathcal{K},[\cdot, \cdot])$ is a Hilbert space, $\llbracket \cdot, \cdot \rrbracket_{\mathcal{K}^{2}}$ is an indefinite inner product. In the following we will say that a linear relation $\Gamma \subset \mathcal{K}^{2} \times \mathcal{H}^{2}$ from $\mathcal{K}^{2}$ to $\mathcal{H}^{2}$ is $\llbracket \cdot, \cdot \rrbracket$-isometric $(\llbracket \cdot, \cdot \rrbracket$-unitary) if $\Gamma$ is an isometric (resp. unitary) relation from $\left(\mathcal{K}^{2}, \llbracket \cdot, \cdot \rrbracket_{\mathcal{K}^{2}}\right)$ to $\left(\mathcal{H}^{2}, \llbracket \cdot, \cdot \rrbracket_{\mathcal{H}^{2}}\right)$. The adjoint of $\Gamma$ will be denoted by $\Gamma^{\llbracket+\rrbracket}$.

\subsection{Definition and basic properties of boundary relations and associated Weyl families}

The notion of boundary relations and associated Weyl families were introduced in [9] for symmetric relations in Hilbert spaces. The definitions and some of the basic properties remain the same in the Krein space case.

Definition 2.1. Let $A \in \widetilde{\mathcal{C}}(\mathcal{K})$ be a symmetric relation in the Krein space $\mathcal{K} . A$ linear relation $\Gamma \subset \mathcal{K}^{2} \times \mathcal{G}^{2}$ is called a boundary relation for $A^{+}$if $\mathcal{G}$ is a Hilbert space, $T:=\operatorname{dom} \Gamma$ is dense in $A^{+}$and $\Gamma$ is $\llbracket \cdot, \rrbracket$-unitary.

Let $A \in \widetilde{\mathcal{C}}(\mathcal{K})$ be a symmetric relation and let $\Gamma \in \widetilde{\mathcal{C}}\left(\mathcal{K}^{2}, \mathcal{G}^{2}\right)$ be a boundary relation for $A^{+}$. Then the first relation in (2.1) implies $A=\operatorname{ker} \Gamma$. The elements in $\Gamma$ will be written in the form $\{\hat{f}, \hat{h}\} \in \Gamma$, where $\hat{f}=\left(\begin{array}{c}f \\ f^{\prime}\end{array}\right) \in \mathcal{K}^{2}$ and $\hat{h}=\left(\begin{array}{c}h \\ h^{\prime}\end{array}\right) \in \mathcal{G}^{2}$. Associated with the boundary relation $\Gamma$ are the relations

$$
\Gamma_{0}:=\{\{\hat{f}, h\} \mid\{\hat{f}, \hat{h}\} \in \Gamma\} \quad \text { and } \quad \Gamma_{1}:=\left\{\left\{\hat{f}, h^{\prime}\right\} \mid\{\hat{f}, \hat{h}\} \in \Gamma\right\} .
$$

We note that $\operatorname{ker} \Gamma_{0}$ and $\operatorname{ker} \Gamma_{1}$ are symmetric relations in $\mathcal{K}$ which in general are not closed.

Definition 2.2. Let $A$ be a closed symmetric relation in $\mathcal{K}$ and let $\Gamma$ be a boundary relation for $A^{+}, T=\operatorname{dom} \Gamma$. The $\gamma$-field $\gamma$ and the Weyl family $\tau$ of the boundary relation $\Gamma$ are defined by

$$
\gamma(\lambda):=\left\{\{h, f\} \mid\{\hat{f}, \hat{h}\} \in \Gamma \text { and } \hat{f} \in \hat{\mathcal{N}}_{\lambda, T}\right\}, \quad \lambda \in \mathbb{C},
$$

and

$$
\tau(\lambda):=\Gamma\left(\hat{\mathcal{N}}_{\lambda, T}\right)=\left\{\hat{h} \mid\{\hat{f}, \hat{h}\} \in \Gamma \text { and } \hat{f} \in \hat{\mathcal{N}}_{\lambda, T}\right\}, \quad \lambda \in \mathbb{C} .
$$


Remark 2.3. In general the values of the Weyl family and the $\gamma$-field corresponding to a boundary relation have nontrivial multivalued parts. For $\{\hat{f}, \hat{h}\}$ with $\hat{f} \in \hat{\mathcal{N}}_{\lambda, T}$, $\lambda \in \mathbb{C}$, the $\llbracket \cdot, \cdot \rrbracket$-unitarity of $\Gamma$ yields

$$
\left(h^{\prime}, h\right)_{\mathcal{G}}-\left(h, h^{\prime}\right)_{\mathcal{G}}=(\lambda-\bar{\lambda})[f, f]_{\mathcal{K}}
$$

In the special case where $\mathcal{K}$ is a Hilbert space, this leads to $\operatorname{ker}\left(\Gamma_{0} \mid \hat{\mathcal{N}}_{\lambda, T}\right)=\{0\}$ for $\lambda \in \mathbb{C} \backslash \mathbb{R}$ and hence, if $A$ is a closed symmetric relation in a Hilbert space $\mathcal{K}$ and $\Gamma$ is a boundary relation for $A^{*}$, then the $\gamma$-field $\gamma$ associated with $\Gamma$ is an operator-valued function on $\mathbb{C} \backslash \mathbb{R}$ which maps $\operatorname{dom} \tau(\lambda)$ onto $\mathcal{N}_{\lambda, T}$, cf. $[9, \S 4.2]$.

Let again $\mathcal{K}$ be a Krein space and let $\mathcal{G}$ be a Hilbert space. Then the bijective transformation

$$
\mathcal{J}: \mathcal{K}^{2} \times \mathcal{G}^{2} \rightarrow(\mathcal{K} \times \mathcal{G})^{2}, \quad\left\{\left(\begin{array}{c}
f \\
f^{\prime}
\end{array}\right),\left(\begin{array}{c}
h \\
h^{\prime}
\end{array}\right)\right\} \mapsto\left\{\left(\begin{array}{c}
f \\
h
\end{array}\right),\left(\begin{array}{c}
f^{\prime} \\
-h^{\prime}
\end{array}\right)\right\}
$$

establishes via $\Gamma \mapsto \mathcal{J}(\Gamma)$ a one-to-one correspondence between the set of $\llbracket \cdot, \cdot \rrbracket$ isometric $\left(\llbracket \cdot, \cdot \rrbracket\right.$-unitary) relations $\Gamma \subset \mathcal{K}^{2} \times \mathcal{G}^{2}$ and the set of symmetric (resp. self-adjoint) relations in $(\mathcal{K} \times \mathcal{G})^{2}$. The mapping $(2.3)$ is called the main transform in [9]. Clearly, if $A$ is closed and symmetric in $\mathcal{K}$ then a relation $\Gamma \subset \mathcal{K}^{2} \times \mathcal{G}^{2}$ with the property $A=\operatorname{ker} \Gamma$ is a boundary relation for $A^{+}$if and only if $\mathcal{J}(\Gamma)$ is self-adjoint. This also implies that for a symmetric relation $A \in \widetilde{\mathcal{C}}(\mathcal{K})$ a boundary relation always exists. The next lemma shows how the Weyl family $\tau$ of a boundary relation $\Gamma$ is connected with the compressed resolvent of $\mathcal{J}(\Gamma)$ onto $\mathcal{G}$. The proof is straightforward and essentially contained in $[9, \S 3]$. We leave the details to the reader.

Lemma 2.4. Let $A$ be a closed symmetric relation in $\mathcal{K}$ and let $\Gamma \in \widetilde{\mathcal{C}}\left(\mathcal{K}^{2}, \mathcal{G}^{2}\right)$ be a boundary relation for $A^{+}$with corresponding Weyl family $\tau$. Define $\mathcal{J}$ as in (2.3) and denote by $P_{\mathcal{G}}$ the orthogonal projection from $\mathcal{K} \times \mathcal{G}$ onto $\mathcal{G}$ and by $\uparrow_{\mathcal{G}}$ the canonical embedding of $\mathcal{G}$ in $\mathcal{K} \times \mathcal{G}$. Then $\tau$ satisfies (i)-(iii).

(i) The formula

$$
P_{\mathcal{G}}(\mathcal{J}(\Gamma)-\lambda)^{-1} \Upsilon_{\mathcal{G}}=-(\tau(\lambda)+\lambda)^{-1}
$$

holds for all $\lambda \in \mathbb{C}$.

(ii) If $\rho(\mathcal{J}(\Gamma))$ is nonempty, then $-(\tau(\lambda)+\lambda)^{-1} \in \mathcal{L}(\mathcal{G})$ for $\lambda \in \rho(\mathcal{J}(\Gamma))$.

(iii) The Weyl family is symmetric with respect to the real line, i.e. $\tau(\bar{\lambda})=\tau(\lambda)^{*}$ for $\lambda \in \mathbb{C} \backslash \mathbb{R}$.

Remark 2.5. The class of Weyl families corresponding to boundary relations for symmetric relations in Hilbert spaces is completely described in [9, Theorem 3.9]. Namely, in the case that $\mathcal{K}$ is a Hilbert space it follows from Lemma 2.4 that the values $\tau(\lambda)$ of the Weyl family $\tau$ are maximal dissipative (maximal accumulative) relations for every $\lambda \in \mathbb{C}_{+}$(resp. $\lambda \in \mathbb{C}_{-}$), and $\tau(\bar{\lambda})=\tau(\lambda)^{*}$ and $-\lambda \in \rho(\tau(\lambda)$ ) holds for all $\lambda \in \mathbb{C} \backslash \mathbb{R}$, i.e. $\tau$ is a so-called Nevanlinna family; we write $\tau \in \widetilde{\mathcal{R}}(\mathcal{G})$. Conversely, by $[9$, Theorem 3.9] each Nevanlinna family $\tau \in \widetilde{\mathcal{R}}(\mathcal{G})$ can be realized 
as the Weyl family of a boundary relation for a symmetric relation in a Hilbert space (see also Corollary 4.4).

\subsection{Boundary triplets for symmetric relations in Krein spaces}

The concept of boundary relations is an extension of the notion of boundary triplets for symmetric relations in Krein and Hilbert spaces, cf. [5, 6, 7] (and e.g. [12, 13, 15] for the Hilbert space case).

Definition 2.6. Let $A$ be a closed symmetric relation in a Krein space $\mathcal{K}$ and let $\Gamma \in \widetilde{\mathcal{C}}\left(\mathcal{K}^{2}, \mathcal{G}^{2}\right)$ be a boundary relation for $A^{+}$with $\Gamma_{0}$ and $\Gamma_{1}$ as in (2.2). If $\Gamma$ is surjective, then $\left\{\mathcal{G}, \Gamma_{0}, \Gamma_{1}\right\}$ is said to be a boundary triplet for $A^{+}$.

Definition 2.6 coincides with the usual definition of a boundary triplet for a symmetric relation since by $(2.1)$ a surjective boundary relation $\Gamma \in \widetilde{\mathcal{C}}\left(\mathcal{K}^{2}, \mathcal{G}^{2}\right)$ is necessarily an operator defined on $A^{+}$and therefore $\Gamma_{0}$ and $\Gamma_{1}$ are operators such that the mapping $\left(\begin{array}{c}\Gamma_{0} \\ \Gamma_{1}\end{array}\right): A^{+} \rightarrow \mathcal{G}^{2}$ is surjective and

$$
\left(f^{\prime}, g\right)_{\mathcal{K}}-\left(f, g^{\prime}\right)_{\mathcal{K}}=\left(\Gamma_{1} \hat{f}, \Gamma_{0} \hat{g}\right)_{\mathcal{G}}-\left(\Gamma_{0} \hat{f}, \Gamma_{1} \hat{g}\right)_{\mathcal{G}}
$$

holds for all $\hat{f}=\left\{f, f^{\prime}\right\}, \hat{g}=\left\{g, g^{\prime}\right\} \in A^{+}$. We briefly recall some important properties of boundary triplets which can be found in e.g. $[5,6,7,12,13]$. Let in the following $A$ be a closed symmetric relation in $\mathcal{K}$ and let $\left\{\mathcal{G}, \Gamma_{0}, \Gamma_{1}\right\}, \Gamma=\left(\begin{array}{l}\Gamma_{0} \\ \Gamma_{1}\end{array}\right)$, be a boundary triplet for $A^{+}$. The mapping $\Gamma$ induces, via

$$
A_{\Theta}:=\Gamma^{-1} \Theta=\left\{\hat{f} \in A^{+} \mid \Gamma \hat{f} \in \Theta\right\},
$$

a bijective correspondence $\Theta \mapsto A_{\Theta}$ between the set of all closed linear relations $\Theta$ in $\mathcal{G}$ and the set of closed extensions $A_{\Theta} \subset A^{+}$of $A$ in $\mathcal{K}$. Furthermore (2.5) establishes a one-to-one correspondence between the closed symmetric (self-adjoint) relations in $\mathcal{G}$ and the closed symmetric (self-adjoint) extensions of $A$ in $\mathcal{K}$. Note, that in particular $A_{0}:=\operatorname{ker} \Gamma_{0}$ and $A_{1}:=\operatorname{ker} \Gamma_{1}$ are self-adjoint extensions of $A$.

Assume now that $\rho\left(A_{0}\right)$ is nonempty. Then for each $\lambda \in \rho\left(A_{0}\right)$ the relation $A^{+}$is the direct sum of $A_{0}$ and $\hat{\mathcal{N}}_{\lambda, A^{+}}$and it follows from Definition 2.2, that for $\lambda \in \rho\left(A_{0}\right)$ the $\gamma$-field $\gamma$ and the Weyl function $M$ of the boundary triplet $\left\{\mathcal{G}, \Gamma_{0}, \Gamma_{1}\right\}$ are given by

$$
\gamma(\lambda)=\pi_{1}\left(\Gamma_{0} \mid \hat{\mathcal{N}}_{\lambda, A^{+}}\right)^{-1} \in \mathcal{L}(\mathcal{G}, \mathcal{K}) \quad \text { and } \quad M(\lambda)=\Gamma_{1}\left(\Gamma_{0} \mid \hat{\mathcal{N}}_{\lambda, A^{+}}\right)^{-1} \in \mathcal{L}(\mathcal{G})
$$

Here $\pi_{1}$ denotes the orthogonal projection onto the first component of $\mathcal{K} \times \mathcal{K}$. The functions $\gamma$ and $M$ are holomorphic on $\rho\left(A_{0}\right)$ and satisfy the relations

$$
\gamma(\lambda)=\left(I+(\lambda-\mu)\left(A_{0}-\lambda\right)^{-1}\right) \gamma(\mu)
$$

and

$$
M(\lambda)-M(\mu)^{*}=(\lambda-\bar{\mu}) \gamma(\mu)^{+} \gamma(\lambda)
$$

for all $\lambda, \mu \in \rho\left(A_{0}\right)$. Moreover

$$
\gamma(\bar{\lambda})^{+} h=\Gamma_{1}\left(\begin{array}{c}
\left(A_{0}-\lambda\right)^{-1} h \\
\left(I+\lambda\left(A_{0}-\lambda\right)^{-1} h\right.
\end{array}\right)
$$


holds for each $h \in \mathcal{K}$ and $\lambda \in \rho\left(A_{0}\right)$

With the help of the Weyl function the spectral properties of the closed extensions $A_{\Theta} \subset A^{+}$of $A$ can be described. Namely, if $\Theta \in \widetilde{\mathcal{C}}(\mathcal{G})$ and $A_{\Theta}$ is the corresponding extension of $A$ via (2.5), then a point $\lambda \in \rho\left(A_{0}\right)$ belongs to $\rho\left(A_{\Theta}\right)$ $\left(\sigma_{i}\left(A_{\Theta}\right), i=p, c, r\right)$ if and only if 0 belongs to $\rho(\Theta-M(\lambda))$ (resp. $\sigma_{i}(\Theta-M(\lambda))$, $i=p, c, r)$ and the well-known formula

$$
\left(A_{\Theta}-\lambda\right)^{-1}=\left(A_{0}-\lambda\right)^{-1}+\gamma(\lambda)(\Theta-M(\lambda))^{-1} \gamma(\bar{\lambda})^{+}
$$

holds for all $\lambda \in \rho\left(A_{0}\right) \cap \rho\left(A_{\Theta}\right)$ (see e.g. [7]).

\section{Generalized resolvents of symmetric relations in Krein spaces}

If $A$ is a closed symmetric operator or relation with equal (possibly infinite) deficiency indices in a Hilbert space $\mathfrak{K}$ and $\left\{\mathcal{G}, \Gamma_{0}, \Gamma_{1}\right\}, A_{0}=\operatorname{ker} \Gamma_{0}$, is a boundary triplet for the adjoint $A^{*} \in \widetilde{\mathcal{C}}(\mathfrak{K})$ with corresponding $\gamma$-field $\gamma$ and Weyl function $M$, then the Krein-Naimark formula

$$
P_{\mathfrak{K}}(\widetilde{A}-\lambda)^{-1} \Gamma_{\mathfrak{K}}=\left(A_{0}-\lambda\right)^{-1}-\gamma(\lambda)(M(\lambda)+\tau(\lambda))^{-1} \gamma(\bar{\lambda})^{*}, \quad \lambda \in \mathbb{C} \backslash \mathbb{R},
$$

establishes a bijective correspondence between the compressed resolvents of minimal self-adjoint extensions $\widetilde{A}$ of $A$ in $\mathfrak{K} \times \mathfrak{H}$, where the exit space $\mathfrak{H}$ is a Hilbert space, and the Nevanlinna families $\tau$, i.e. the Weyl families of boundary relations of symmetric relations in Hilbert spaces (see e.g. $[8,10,11,12,13,24,27])$. In this section we prove some variants of (3.1) for the case that $\mathfrak{K}$ and $\mathfrak{H}$ are Krein spaces. Other indefinite generalizations of (3.1) can be found in [3, 4, 5, 6, 7, 21, 22].

\subsection{The case of a Krein space as exit space}

In the next theorem we show, roughly speaking, that a correspondence of the form (3.1) exists also between the compressed resolvents of the self-adjoint extensions of a symmetric relation in a Krein space $\mathcal{K}$ and the Weyl families of boundary relations of symmetric relations acting in Krein spaces $\mathcal{H}$. The idea of the proof is based on the coupling method from [8].

Theorem 3.1. Let $\mathcal{K}$ and $\mathcal{H}$ be Krein spaces, let $A \in \widetilde{\mathcal{C}}(\mathcal{K})$ be a symmetric relation and let $\left\{\mathcal{G}, \Gamma_{0}, \Gamma_{1}\right\}$ be a boundary triplet for $A^{+}$with corresponding $\gamma$-field $\gamma$ and Weyl function $M$. Let $A_{0}=\operatorname{ker} \Gamma_{0}$ and assume that $\rho\left(A_{0}\right)$ is nonempty.

(i) If $\widetilde{A} \in \widetilde{\mathcal{C}}(\mathcal{K} \times \mathcal{H})$ is a self-adjoint extension of $A$ and for some $\lambda_{0} \in \rho\left(A_{0}\right)$ $\left.P_{\mathcal{K}}\left(\widetilde{A}-\lambda_{0}\right)^{-1}\right|_{\mathcal{K}} \in \mathcal{L}(\mathcal{K})$, then there exists a boundary relation $\Gamma^{\prime} \in \widetilde{\mathcal{C}}\left(\mathcal{H}^{2}, \mathcal{G}^{2}\right)$ such that the corresponding Weyl family $\tau$ satisfies (a) and (b).

(a) If $\lambda \in \rho\left(A_{0}\right)$, then $(M(\lambda)+\tau(\lambda))^{-1} \in \mathcal{L}(\mathcal{G})$ if and only if $P_{\mathcal{K}}(\widetilde{A}-\lambda)^{-1} \Gamma_{\mathcal{K}} \in \mathcal{L}(\mathcal{K})$.

(b) The formula

$$
P_{\mathcal{K}}(\widetilde{A}-\lambda)^{-1}\left\lceil_{\mathcal{K}}=\left(A_{0}-\lambda\right)^{-1}-\gamma(\lambda)(M(\lambda)+\tau(\lambda))^{-1} \gamma(\bar{\lambda})^{+}\right.
$$


holds for all $\lambda \in \rho\left(A_{0}\right)$ such that $(M(\lambda)+\tau(\lambda))^{-1} \in \mathcal{L}(\mathcal{G})$.

(ii) If $\Gamma^{\prime} \in \widetilde{\mathcal{C}}\left(\mathcal{H}^{2}, \mathcal{G}^{2}\right)$ is a boundary relation with corresponding Weyl family $\tau$ and $\left(M\left(\lambda_{0}\right)+\tau\left(\lambda_{0}\right)\right)^{-1} \in \mathcal{L}(\mathcal{G})$ for some $\lambda_{0} \in \rho\left(A_{0}\right)$, then there exists a self-adjoint extension $\widetilde{A} \in \widetilde{\mathcal{C}}(\mathcal{K} \times \mathcal{H})$ of $A$ such that (a) and (b) are satisfied.

Proof. (i) 1. The proof of assertion (i) is organized in 4 steps. Let $\mathcal{H}$ be a Krein space and let $\widetilde{A}$ be a self-adjoint extension of $A$ in $\mathcal{K} \times \mathcal{H}$. We do not exclude the case of a canonical self-adjoint extension $\widetilde{A}$, that is, $\mathcal{H}=\{0\}$. It is not difficult to see that the closed linear relations

$$
\begin{aligned}
& S_{0}:=\left\{\left\{f_{0}, f_{0}^{\prime}\right\} \in \mathcal{K}^{2} \mid\left\{\left(\begin{array}{c}
f_{0} \\
0
\end{array}\right),\left(\begin{array}{c}
f_{0}^{\prime} \\
0
\end{array}\right)\right\} \in \widetilde{A}\right\}, \\
& S_{1}:=\left\{\left\{f_{1}, f_{1}^{\prime}\right\} \in \mathcal{H}^{2} \mid\left\{\left(\begin{array}{c}
0 \\
f_{1}
\end{array}\right),\left(\begin{array}{c}
0 \\
f_{1}^{\prime}
\end{array}\right)\right\} \in \widetilde{A}\right\}
\end{aligned}
$$

are symmetric in $\mathcal{K}$ and $\mathcal{H}$, respectively. The same arguments as in the Hilbert space case (see [9, Proposition 2.12]) imply that the closures of the linear relations

$$
\begin{aligned}
& T_{0}:=\left\{\left\{f_{0}, f_{0}^{\prime}\right\} \in \mathcal{K}^{2} \mid\left\{\left(\begin{array}{l}
f_{0} \\
f_{1}
\end{array}\right),\left(\begin{array}{l}
f_{0}^{\prime} \\
f_{1}^{\prime}
\end{array}\right)\right\} \in \widetilde{A}\right\}, \\
& T_{1}:=\left\{\left\{f_{1}, f_{1}^{\prime}\right\} \in \mathcal{H}^{2} \mid\left\{\left(\begin{array}{l}
f_{0} \\
f_{1}
\end{array}\right),\left(\begin{array}{l}
f_{0}^{\prime} \\
f_{1}^{\prime}
\end{array}\right)\right\} \in \widetilde{A}\right\}
\end{aligned}
$$

coincide with $S_{0}^{+}$and $S_{1}^{+}$, i.e. $S_{i} \subset T_{i} \subset \bar{T}_{i}=S_{i}^{+}$holds for $i=1,2$. Note also that $S_{0}$ is an extension of the symmetric relation $A$.

2. In this step we show that

$$
\Gamma^{\prime}:=\left\{\left\{\hat{f}_{1},\left(\begin{array}{c}
\Gamma_{0} \hat{f}_{0} \\
-\Gamma_{1} \hat{f}_{0}
\end{array}\right)\right\} \in \mathcal{H}^{2} \times \mathcal{G}^{2} \mid\left\{\left(\begin{array}{c}
f_{0} \\
f_{1}
\end{array}\right),\left(\begin{array}{c}
f_{0}^{\prime} \\
f_{1}^{\prime}
\end{array}\right)\right\} \in \widetilde{A}\right\}
$$

is a boundary relation for $S_{1}^{+}$.

¿From the definition of $\Gamma^{\prime}$ we immediately get that dom $\Gamma^{\prime}$ is dense in $S_{1}^{+}$. In order to verify that the linear relation $\Gamma^{\prime} \subset \mathcal{H}^{2} \times \mathcal{G}^{2}$ is $\llbracket \cdot, \cdot \rrbracket$-unitary let

$$
\left\{\left(\begin{array}{c}
\Gamma_{0} \hat{f}_{0} \\
-\Gamma_{1} \hat{f}_{0}
\end{array}\right), \hat{f}_{1}\right\} \in \Gamma^{\prime-1} \text { and } \quad\left\{\hat{g}_{1},\left(\begin{array}{c}
\Gamma_{0} \hat{g}_{0} \\
-\Gamma_{1} \hat{g}_{0}
\end{array}\right)\right\} \in \Gamma^{\prime} .
$$

As $\widetilde{A}$ is self-adjoint in $\mathcal{K} \times \mathcal{H}$ we have

and hence

$$
\left[\left(\begin{array}{l}
f_{0} \\
f_{1}
\end{array}\right),\left(\begin{array}{l}
g_{0}^{\prime} \\
g_{1}^{\prime}
\end{array}\right)\right]_{\mathcal{K} \times \mathcal{H}}=\left[\left(\begin{array}{l}
f_{0}^{\prime} \\
f_{1}^{\prime}
\end{array}\right),\left(\begin{array}{l}
g_{0} \\
g_{1}
\end{array}\right)\right]_{\mathcal{K} \times \mathcal{H}}
$$

$$
\llbracket\left(\begin{array}{c}
\Gamma_{0} \hat{f}_{0} \\
-\Gamma_{1} \hat{f}_{0}
\end{array}\right),\left(\begin{array}{c}
\Gamma_{0} \hat{g}_{0} \\
-\Gamma_{1} \hat{g}_{0}
\end{array}\right) \rrbracket_{\mathcal{G}^{2}}=i\left(\left[f_{0}^{\prime}, g_{0}\right]_{\mathcal{K}}-\left[f_{0}, g_{0}^{\prime}\right]_{\mathcal{K}}\right)=\llbracket \hat{f}_{1}, \hat{g}_{1} \rrbracket_{\mathcal{H}^{2}}
$$

implies that $\Gamma^{\prime}$ is $\llbracket \cdot, \cdot \rrbracket$-isometric, i.e. $\Gamma^{\prime-1} \subset \Gamma^{\prime \llbracket+\rrbracket}$. Conversely, let $\left\{\hat{h}, \hat{f}_{1}\right\} \in \Gamma^{\prime} \llbracket+\rrbracket$ and choose $\hat{f}_{0} \in A^{+}$such that $\hat{h}=\left(\begin{array}{c}\Gamma_{0} \hat{f}_{0} \\ -\Gamma_{1} \hat{f}_{0}\end{array}\right)$. Then $\left\{\left(\begin{array}{c}f_{0} \\ f_{1}\end{array}\right),\left(\begin{array}{c}f_{0}^{\prime} \\ f_{1}^{\prime}\end{array}\right)\right\}$ belongs to $\widetilde{A}=\widetilde{A}^{+}$ 
since for an arbitrary $\left\{\left(\begin{array}{c}g_{0} \\ g_{1}\end{array}\right),\left(\begin{array}{c}g_{0}^{\prime} \\ g_{1}^{\prime}\end{array}\right)\right\} \in \widetilde{A}$ we have $\left\{\hat{g}_{1},\left(\begin{array}{c}\Gamma_{0} \hat{g}_{0} \\ -\Gamma_{1} \hat{g}_{0}\end{array}\right)\right\} \in \Gamma^{\prime}$ and hence $\left\{\hat{h}, \hat{f}_{1}\right\} \in \Gamma^{\prime} \llbracket+\rrbracket$ and the choice of $\hat{h}$ imply

$$
\begin{array}{r}
{\left[\left(\begin{array}{c}
f_{0} \\
f_{1}
\end{array}\right),\left(\begin{array}{c}
g_{0}^{\prime} \\
g_{1}^{\prime}
\end{array}\right)\right]_{\mathcal{K} \times \mathcal{H}}-\left[\left(\begin{array}{c}
f_{0}^{\prime} \\
f_{1}^{\prime}
\end{array}\right),\left(\begin{array}{c}
g_{0} \\
g_{1}
\end{array}\right)\right]_{\mathcal{K} \times \mathcal{H}}=-i\left(\llbracket \hat{f}_{0}, \hat{g}_{0} \rrbracket_{\mathcal{K}^{2}}+\left[\hat{f}_{1}, \hat{g}_{1} \rrbracket_{\mathcal{H}^{2}}\right)\right.} \\
=-i\left(\llbracket\left(\begin{array}{c}
\Gamma_{0} \hat{f}_{0} \\
\Gamma_{1} \hat{f}_{0}
\end{array}\right),\left(\begin{array}{c}
\Gamma_{0} \hat{g}_{0} \\
\Gamma_{1} \hat{g}_{0}
\end{array}\right) \rrbracket_{\mathcal{G}^{2}}+\llbracket \hat{h},\left(\begin{array}{c}
\Gamma_{0} \hat{g}_{0} \\
-\Gamma_{1} \hat{g}_{0}
\end{array}\right) \rrbracket_{\mathcal{G}^{2}}\right)=0 .
\end{array}
$$

Hence $\left\{\hat{f}_{1}, \hat{h}\right\}$ belongs to $\Gamma^{\prime}$ and this gives $\Gamma^{\prime \llbracket+\rrbracket} \subset \Gamma^{\prime-1}$. We have shown that $\Gamma^{\prime}$ is a boundary relation for $S_{1}^{+}$.

3. Let $\lambda \in \rho\left(A_{0}\right)$ such that $P_{\mathcal{K}}(\widetilde{A}-\lambda)^{-1}\left\lceil_{\mathcal{K}} \in \mathcal{L}(\mathcal{K})\right.$ and denote the Weyl family of $\Gamma^{\prime}$ by $\tau$. We check that

$$
(M(\lambda)+\tau(\lambda))^{-1}=\left\{\left(\begin{array}{c}
\Gamma_{1} \hat{f}_{\lambda}+h^{\prime} \\
\Gamma_{0} \hat{f}_{\lambda}
\end{array}\right) \in \mathcal{G}^{2} \mid \hat{f}_{\lambda} \in \hat{\mathcal{N}}_{\lambda, A^{+}} \text {and }\left(\begin{array}{c}
\Gamma_{0} \hat{f}_{\lambda} \\
h^{\prime}
\end{array}\right) \in \tau(\lambda)\right\}
$$

is a bounded operator defined on $\mathcal{G}$.

If $\Gamma_{1} \hat{f}_{\lambda}+h^{\prime}=0$ then $\left(\begin{array}{c}\Gamma_{0} \hat{f}_{\lambda} \\ -\Gamma_{1} \hat{f}_{\lambda}\end{array}\right) \in \tau(\lambda)$ and hence there exists $\hat{f}_{1} \in \hat{\mathcal{N}}_{\lambda, T_{1}}$ such that $\left\{\hat{f}_{1},\left(\begin{array}{c}\Gamma_{0} \hat{f}_{\lambda} \\ -\Gamma_{1} \hat{f}_{\lambda}\end{array}\right)\right\} \in \Gamma^{\prime}$. By $(3.3)\left\{\left(\begin{array}{c}f_{\lambda} \\ f_{1}\end{array}\right),\left(\begin{array}{c}\lambda f_{\lambda} \\ \lambda f_{1}\end{array}\right)\right\} \in \widetilde{A}$ and this implies that $\left\{0, f_{\lambda}\right\}$ belongs to

$$
\left.P_{\mathcal{K}}(\widetilde{A}-\lambda)^{-1}\right|_{\mathcal{K}}=\left\{\left\{f_{0}^{\prime}-\lambda f_{0}, f_{0}\right\} \in \mathcal{K}^{2} \mid\left\{\left(\begin{array}{l}
f_{0} \\
f_{1}
\end{array}\right),\left(\begin{array}{l}
f_{0}^{\prime} \\
f_{1}^{\prime}
\end{array}\right)\right\} \in \widetilde{A} \text { and } \hat{f}_{1} \in \hat{\mathcal{N}}_{\lambda, T_{1}}\right\} .
$$

Therefore $f_{\lambda}=0$, i.e. $\Gamma_{0} \hat{f}_{\lambda}=0$ and $(M(\lambda)+\tau(\lambda))^{-1}$ is an operator. Next we show

$$
\operatorname{dom}(M(\lambda)+\tau(\lambda))^{-1}=\mathcal{G} .
$$

Let $g \in \mathcal{G}$ and choose $\hat{f}_{0} \in A^{+}$such that $\left(\begin{array}{c}\Gamma_{0} \hat{f}_{0} \\ -\Gamma_{1} \hat{f}_{0}\end{array}\right)=\left(\begin{array}{l}0 \\ g\end{array}\right)$. By our assumption $\operatorname{dom}\left(P_{\mathcal{K}}(\widetilde{A}-\lambda)^{-1}\lceil\mathcal{K})=\mathcal{K}\right.$ there exists $\hat{f} \in A^{+}$and $\hat{f}_{1} \in \hat{\mathcal{N}}_{\lambda, T_{1}}$ such that $\left\{\left(\begin{array}{c}f \\ f_{1}\end{array}\right),\left(\begin{array}{c}f^{\prime} \\ \lambda f_{1}\end{array}\right)\right\} \in \widetilde{A}$ and $f^{\prime}-\lambda f=f_{0}^{\prime}-\lambda f_{0}$. Hence $\hat{f}_{\lambda}:=\hat{f}-\hat{f}_{0} \in \hat{\mathcal{N}}_{\lambda, A^{+}}$and $\left\{\hat{f}_{1},\left(\begin{array}{c}\Gamma_{0} \hat{f} \\ -\Gamma_{1} \hat{f}\end{array}\right)\right\} \in \Gamma^{\prime}$, i.e. $\left(\begin{array}{c}\Gamma_{0} \hat{f} \\ -\Gamma_{1} \hat{f}\end{array}\right) \in \tau(\lambda)$. Setting $h^{\prime}:=-\Gamma_{1} \hat{f}$ we find $\left(\begin{array}{c}\Gamma_{0} \hat{f}_{\lambda} \\ h^{\prime}\end{array}\right) \in \tau(\lambda)$ and

$$
\Gamma_{1} \hat{f}_{\lambda}+h^{\prime}=\Gamma_{1}\left(\hat{f}-\hat{f}_{0}\right)+h^{\prime}=-\Gamma_{1} \hat{f}_{0}=g
$$

that is $g \in \operatorname{dom}(M(\lambda)+\tau(\lambda))^{-1}$. Finally $M(\lambda) \in \mathcal{L}(\mathcal{G})$ and the fact that $\tau(\lambda)$ is closed imply that $(M(\lambda)+\tau(\lambda))^{-1}$ is closed and therefore $(M(\lambda)+\tau(\lambda))^{-1} \in \mathcal{L}(\mathcal{G})$.

4. Let now $\lambda \in \rho\left(A_{0}\right)$ such that $(M(\lambda)+\tau(\lambda))^{-1} \in \mathcal{L}(\mathcal{G})$ holds. We prove in this step that $P_{\mathcal{K}}(\widetilde{A}-\lambda)^{-1} \mid \mathcal{K} \in \mathcal{L}(\mathcal{K})$ has the form (3.2).

Let $k \in \mathcal{K}$ and $f_{\lambda}:=-\gamma(\lambda)\left((M(\lambda)+\tau(\lambda))^{-1} \gamma(\bar{\lambda})^{+} k \in \mathcal{N}_{\lambda, A^{+}}\right.$and define $\hat{f}_{0} \in A^{+}$by

$$
\hat{f}_{0}=\left(\begin{array}{c}
f_{0} \\
f_{0}^{\prime}
\end{array}\right):=\left(\begin{array}{c}
\left(A_{0}-\lambda\right)^{-1} k \\
k+\lambda\left(A_{0}-\lambda\right)^{-1} k
\end{array}\right)+\left(\begin{array}{c}
f_{\lambda} \\
\lambda f_{\lambda}
\end{array}\right) .
$$


We have

$$
f_{0}^{\prime}-\lambda f_{0}=k \quad \text { and } \quad f_{0}=\left(A_{0}-\lambda\right)^{-1} k-\gamma(\lambda)\left((M(\lambda)+\tau(\lambda))^{-1} \gamma(\bar{\lambda})^{+} k .\right.
$$

Therefore, by the form of $P_{\mathcal{K}}(\widetilde{A}-\lambda)^{-1} \uparrow_{\mathcal{K}}$ it remains to show that there exists $\hat{f}_{1} \in \hat{\mathcal{N}}_{\lambda, T_{1}}$ with $\left\{\left(\begin{array}{c}f_{0} \\ f_{1}\end{array}\right),\left(\begin{array}{c}f_{0}^{\prime} \\ f_{1}^{\prime}\end{array}\right)\right\} \in \widetilde{A}$. First of all

$$
\Gamma_{0} \hat{f}_{\lambda}=-(M(\lambda)+\tau(\lambda))^{-1} \gamma(\bar{\lambda})^{+} k
$$

implies

$$
\left(\begin{array}{c}
\Gamma_{0} \hat{f}_{\lambda} \\
-\gamma(\bar{\lambda})^{+} k
\end{array}\right) \in(M(\lambda)+\tau(\lambda))=\left\{\left(\begin{array}{c}
h \\
M(\lambda) h+h^{\prime}
\end{array}\right) \mid \hat{h} \in \tau(\lambda)\right\}
$$

and this gives

$$
\left(\begin{array}{c}
\Gamma_{0} \hat{f}_{\lambda} \\
-\gamma(\bar{\lambda})^{+} k-\Gamma_{1} \hat{f}_{\lambda}
\end{array}\right) \in \tau(\lambda)=\Gamma^{\prime}\left(\hat{\mathcal{N}}_{\lambda, T_{1}}\right)
$$

Hence there exists $\hat{f}_{1} \in \hat{\mathcal{N}}_{\lambda, T_{1}}$ such that

$$
\left\{\hat{f}_{1},\left(\begin{array}{c}
\Gamma_{0} \hat{f}_{\lambda} \\
-\gamma(\bar{\lambda})^{+} k-\Gamma_{1} \hat{f}_{\lambda}
\end{array}\right)\right\} \in \Gamma^{\prime}
$$

and from (3.4) and (2.6) we obtain $\Gamma_{0} \hat{f}_{0}=\Gamma_{0} \hat{f}_{\lambda}$ and $\Gamma_{1} \hat{f}_{0}=\gamma(\bar{\lambda})^{+} k+\Gamma_{1} \hat{f}_{\lambda}$. Therefore $\left\{\hat{f}_{1},\left(\begin{array}{c}\Gamma_{0} \hat{f}_{0} \\ -\Gamma_{1} \hat{f}_{0}\end{array}\right)\right\}$ belongs to $\Gamma^{\prime}$ and thus by $(3.3)\left\{\left(\begin{array}{c}f_{0} \\ f_{1}\end{array}\right),\left(\begin{array}{c}f_{0}^{\prime} \\ f_{1}^{\prime}\end{array}\right)\right\} \in \widetilde{A}$. This completes the proof of assertion (i).

We prove assertion (ii). Let $\Gamma^{\prime} \in \widetilde{\mathcal{C}}\left(\mathcal{H}^{2}, \mathcal{G}^{2}\right)$ be a boundary relation with corresponding Weyl family $\tau$. We claim that

$$
\widetilde{A}:=\left\{\left\{\left(\begin{array}{l}
f_{0} \\
f_{1}
\end{array}\right),\left(\begin{array}{l}
f_{0}^{\prime} \\
f_{1}^{\prime}
\end{array}\right)\right\} \in(\mathcal{K} \times \mathcal{H})^{2} \mid\left\{\hat{f}_{1},\left(\begin{array}{c}
\Gamma_{0} \hat{f}_{0} \\
-\Gamma_{1} \hat{f}_{0}
\end{array}\right)\right\} \in \Gamma^{\prime}\right\}
$$

is a self-adjoint extension of $A$. In fact, for $\left\{\left(\begin{array}{c}f_{0} \\ f_{1}\end{array}\right),\left(\begin{array}{c}f_{0}^{\prime} \\ f_{1}^{\prime}\end{array}\right)\right\},\left\{\left(\begin{array}{c}g_{0} \\ g_{1}\end{array}\right),\left(\begin{array}{c}g_{0}^{\prime} \\ g_{1}^{\prime}\end{array}\right)\right\} \in \widetilde{A}$ we have $\left\{\hat{f}_{1},\left(\begin{array}{c}\Gamma_{0} \hat{f}_{0} \\ -\Gamma_{1} \hat{f}_{0}\end{array}\right)\right\},\left\{\hat{g}_{1},\left(\begin{array}{c}\Gamma_{0} \hat{g}_{0} \\ -\Gamma_{0} \hat{g}_{1}\end{array}\right)\right\} \in \Gamma^{\prime}$ and the $\llbracket \cdot, \cdot \rrbracket$-isometry of $\Gamma^{\prime}$ implies

$$
\begin{array}{r}
{\left[\left(\begin{array}{l}
f_{0} \\
f_{1}
\end{array}\right),\left(\begin{array}{l}
g_{0}^{\prime} \\
g_{1}^{\prime}
\end{array}\right)\right] \mathcal{K} \times \mathcal{H}-\left[\left(\begin{array}{c}
f_{0}^{\prime} \\
f_{1}^{\prime}
\end{array}\right),\left(\begin{array}{l}
g_{0} \\
g_{1}
\end{array}\right)\right]_{\mathcal{K} \times \mathcal{H}}=-i\left(\llbracket \hat{f}_{0}, \hat{g}_{0} \rrbracket_{\mathcal{K}^{2}}+\llbracket \hat{f}_{1}, \hat{g}_{1} \rrbracket_{\mathcal{H}^{2}}\right)} \\
=-i\left(\llbracket\left(\begin{array}{c}
\Gamma_{0} \hat{f}_{0} \\
\Gamma_{1} \hat{f}_{0}
\end{array}\right),\left(\begin{array}{c}
\Gamma_{0} \hat{g}_{0} \\
\Gamma_{1} \hat{g}_{0}
\end{array}\right) \rrbracket_{\mathcal{G}^{2}}+\llbracket\left(\begin{array}{c}
\Gamma_{0} \hat{f}_{0} \\
-\Gamma_{1} \hat{f}_{0}
\end{array}\right),\left(\begin{array}{c}
\Gamma_{0} \hat{g}_{0} \\
-\Gamma_{1} \hat{g}_{0}
\end{array}\right) \rrbracket_{\mathcal{G}^{2}}\right)=0,
\end{array}
$$

that is, $\widetilde{A}$ is symmetric. Let now $\left\{\left(\begin{array}{c}f_{0} \\ f_{1}\end{array}\right),\left(\begin{array}{c}f_{0}^{\prime} \\ f_{1}^{\prime}\end{array}\right)\right\} \in \widetilde{A}^{+}$. We show that $\left\{\left(\begin{array}{c}f_{0} \\ f_{1}\end{array}\right),\left(\begin{array}{l}f_{0}^{\prime} \\ f_{1}^{\prime}\end{array}\right)\right\}$ belongs to $\widetilde{A}$. First of all we have

$$
\llbracket \hat{f}_{0}, \hat{g}_{0} \rrbracket_{\mathcal{K}^{2}}=-\llbracket \hat{f}_{1}, \hat{g}_{1} \rrbracket_{\mathcal{H}^{2}} \quad \text { for all } \quad\left\{\left(\begin{array}{c}
g_{0} \\
g_{1}
\end{array}\right),\left(\begin{array}{c}
g_{0}^{\prime} \\
g_{1}^{\prime}
\end{array}\right)\right\} \in \widetilde{A} .
$$


If $\hat{g}_{0} \in A=\operatorname{ker} \Gamma_{0} \cap \operatorname{ker} \Gamma_{1}$ we conclude $\left\{\left(\begin{array}{c}g_{0} \\ 0\end{array}\right),\left(\begin{array}{c}g_{0}^{\prime} \\ 0\end{array}\right)\right\} \in \widetilde{A}$ from $\{0,0\} \in \Gamma^{\prime}$ and (3.6) yields in this case $\hat{f}_{0} \in A^{+}$. By (3.5) it remains to check $\left\{\hat{f}_{1},\left(\begin{array}{c}\Gamma_{0} \hat{f}_{0} \\ -\Gamma_{1} \hat{f}_{0}\end{array}\right)\right\} \in \Gamma^{\prime}$. For this let $\left\{\hat{g}_{1}, \hat{k}\right\} \in \Gamma^{\prime}$ and choose $\hat{g}_{0} \in A^{+}$such that $\hat{k}=\left(\begin{array}{c}\Gamma_{0} \hat{g}_{0} \\ -\Gamma_{1} \hat{g}_{0}\end{array}\right)$. By (3.5) $\left\{\left(\begin{array}{l}g_{0} \\ g_{1}\end{array}\right),\left(\begin{array}{c}g_{0}^{\prime} \\ g_{1}^{\prime}\end{array}\right)\right\}$ belongs to $\widetilde{A}$ and (3.6) implies

$$
\llbracket \hat{f}_{1}, \hat{g}_{1} \rrbracket_{\mathcal{H}^{2}}=-\llbracket \hat{f}_{0}, \hat{g}_{0} \rrbracket_{\mathcal{K}^{2}}=-\llbracket\left(\begin{array}{c}
\Gamma_{0} \hat{f}_{0} \\
\Gamma_{1} \hat{f}_{0}
\end{array}\right),\left(\begin{array}{c}
\Gamma_{0} \hat{g}_{0} \\
\Gamma_{1} \hat{g}_{0}
\end{array}\right) \rrbracket_{\mathcal{G}^{2}}=\llbracket\left(\begin{array}{c}
\Gamma_{0} \hat{f}_{0} \\
-\Gamma_{1} \hat{f}_{0}
\end{array}\right), \hat{k} \rrbracket_{\mathcal{G}^{2}} .
$$

Therefore $\left\{\left(\begin{array}{c}\Gamma_{0} \hat{f}_{0} \\ -\Gamma_{1} \hat{f}_{0}\end{array}\right), \hat{f}_{1}\right\} \in \Gamma^{\prime \llbracket+\rrbracket}=\Gamma^{\prime-1}$ and this gives $\left\{\left(\begin{array}{c}f_{0} \\ f_{1}\end{array}\right),\left(\begin{array}{c}f_{0}^{\prime} \\ f_{1}^{\prime}\end{array}\right)\right\} \in \widetilde{A}$. We have shown that $\widetilde{A}$ is self-adjoint. Moreover it is not difficult to see that $\widetilde{A} \in \widetilde{\mathcal{C}}(\mathcal{K} \times \mathcal{H})$ is an extension of $A$.

If $\widetilde{A}$ is defined by (3.5) then the boundary relation $\Gamma^{\prime}$ can be written in the form

$$
\Gamma^{\prime}=\left\{\left\{\hat{f}_{1},\left(\begin{array}{c}
\Gamma_{0} \hat{f}_{0} \\
-\Gamma_{1} \hat{f}_{0}
\end{array}\right)\right\} \in \mathcal{H}^{2} \times \mathcal{G}^{2} \mid\left\{\left(\begin{array}{c}
f_{0} \\
f_{1}
\end{array}\right),\left(\begin{array}{c}
f_{0}^{\prime} \\
f_{1}^{\prime}
\end{array}\right)\right\} \in \widetilde{A}\right\} .
$$

It was shown in step 2 of the proof of (i) that $\Gamma^{\prime}$ is a boundary relation for $S_{1}^{+}$(see step 1) and by step 3 and 4 the assertions (a) and (b) of (i) hold. We have proved Theorem 3.1.

\subsection{The case of a Hilbert space as exit space}

We are now concerned with the situation that the exit space $\mathcal{H}$ is a Hilbert space. Under this additional assumption assertion (a) of the previous theorem can be improved.

Theorem 3.2. Let $\mathcal{K}$ be a Krein space and let $\mathcal{H}$ be a Hilbert space, let $A \in \widetilde{\mathcal{C}}(\mathcal{K})$ be a symmetric relation and let $\left\{\mathcal{G}, \Gamma_{0}, \Gamma_{1}\right\}$ be a boundary triplet for $A^{+}$with corresponding $\gamma$-field $\gamma$ and Weyl function $M$. Let $A_{0}=\operatorname{ker} \Gamma_{0}$ and assume that $\rho\left(A_{0}\right)$ is nonempty.

(i) If $\widetilde{A} \in \widetilde{\mathcal{C}}(\mathcal{K} \times \mathcal{H})$ is a self-adjoint extension of $A$ and $P_{\mathcal{K}}\left(\widetilde{A}-\lambda_{0}\right)^{-1} \uparrow_{\mathcal{K}} \in \mathcal{L}(\mathcal{K})$ holds for some $\lambda_{0} \in \rho\left(A_{0}\right)$, then there exists a Nevanlinna family $\tau \in \widetilde{\mathcal{R}}(\mathcal{G})$ such that (a) and (b) hold.

(a) If $\lambda \in \rho\left(A_{0}\right)$, then $(M(\lambda)+\tau(\lambda))^{-1} \in \mathcal{L}(\mathcal{G})$ if and only if $\lambda \in \rho(\widetilde{A})$.

(b) The formula

$$
P_{\mathcal{K}}(\widetilde{A}-\lambda)^{-1}\left\lceil_{\mathcal{K}}=\left(A_{0}-\lambda\right)^{-1}-\gamma(\lambda)(M(\lambda)+\tau(\lambda))^{-1} \gamma(\bar{\lambda})^{+}\right.
$$

holds for all $\lambda \in \rho\left(A_{0}\right) \cap \rho(\widetilde{A})$.

(ii) If $\tau \in \widetilde{\mathcal{R}}(\mathcal{G})$ is a Nevanlinna family and $\left(M\left(\lambda_{0}\right)+\tau\left(\lambda_{0}\right)\right)^{-1} \in \mathcal{L}(\mathcal{G})$ for some $\lambda_{0} \in \rho\left(A_{0}\right)$, then there exists a self-adjoint extension $\widetilde{A} \in \widetilde{\mathcal{C}}(\mathcal{K} \times \mathcal{H})$ of $A$ such that (a) and (b) are satisfied. 
Proof. Since the class of Nevanlinna families $\widetilde{\mathcal{R}}(\mathcal{G})$ coincides with the class of Weyl families of boundary relations in Hilbert spaces (see [9, Theorem 3.9], Remark 2.5 and Corollary 4.4) the assertions of Theorem 3.2 follow immediately from Theorem 3.1 if we show that each point $\lambda \in \rho\left(A_{0}\right)$ with the property $(M(\lambda)+\tau(\lambda))^{-1} \in \mathcal{L}(\mathcal{G})$ belongs to $\rho(\widetilde{A})$.

Define $\Gamma^{\prime} \in \widetilde{\mathcal{C}}\left(\mathcal{H}^{2}, \mathcal{G}^{2}\right)$ as in $(3.3)$, let $\tau$ and $\gamma^{\prime}$ be the corresponding Weyl family and $\gamma$-field and assume that $\lambda \in \rho\left(A_{0}\right)$ is chosen such that $(M(\lambda)+\tau(\lambda))^{-1} \in \mathcal{L}(\mathcal{G})$. In order to show that $\widetilde{A}-\lambda$ is injective, let

$$
\left\{\left(\begin{array}{l}
f_{0} \\
f_{1}
\end{array}\right),\left(\begin{array}{l}
f_{0}^{\prime} \\
f_{1}^{\prime}
\end{array}\right)\right\} \in \widetilde{A} \text { such that } f_{0}^{\prime}-\lambda f_{0}=0 \text { and } f_{1}^{\prime}-\lambda f_{1}=0
$$

i.e. $\hat{f}_{0} \in \mathcal{N}_{\lambda, A^{+}}$and $\hat{f}_{1} \in \mathcal{N}_{\lambda, T_{1}}$. Writing $\widetilde{A}$ in the form (3.5) and setting $h:=\Gamma_{0} \hat{f}_{0}$ we conclude

$$
\left(\begin{array}{c}
h \\
-M(\lambda) h
\end{array}\right)=\left(\begin{array}{c}
\Gamma_{0} \hat{f}_{0} \\
-\Gamma_{1} \hat{f}_{0}
\end{array}\right) \in \Gamma^{\prime}\left(\mathcal{N}_{\lambda, T_{1}}\right)=\tau(\lambda)
$$

and therefore $\left(\begin{array}{c}h \\ 0\end{array}\right) \in M(\lambda)+\tau(\lambda)$. From $(M(\lambda)+\tau(\lambda))^{-1} \in \mathcal{L}(\mathcal{G})$ we now get $h=0$ and since both $\gamma(\lambda)$ and $\gamma^{\prime}(\lambda)$ are operators (cf. Remark 2.3) here we obtain $f_{0}=\gamma(\lambda) h=0$ and $f_{1}=\gamma^{\prime}(\lambda) h=0$, that is, $\operatorname{ker}(\widetilde{A}-\lambda)=\{0\}$.

In order to show the surjectivity of $\widetilde{A}-\lambda$ we construct elements $\hat{\mathfrak{f}}_{0} \in A^{+}$and $\hat{\mathfrak{f}}_{1} \in T_{1}$ with

$$
\left\{\hat{\mathfrak{f}}_{1},\left(\begin{array}{c}
\Gamma_{0} \hat{\mathfrak{f}}_{0} \\
-\Gamma_{1} \hat{\mathfrak{f}}_{0}
\end{array}\right)\right\} \in \Gamma^{\prime} \text { and }\left(\begin{array}{c}
\mathfrak{f}_{0}^{\prime}-\lambda \mathfrak{f}_{0} \\
\mathfrak{f}_{1}^{\prime}-\lambda \mathfrak{f}_{1}
\end{array}\right)=\left(\begin{array}{c}
g_{0} \\
g_{1}
\end{array}\right)
$$

for an arbitrary $\left(\begin{array}{l}g_{0} \\ g_{1}\end{array}\right) \in \mathcal{K} \times \mathcal{H}$. First of all choose $\hat{f}_{0} \in A_{0}$ such that $f_{0}^{\prime}-\lambda f_{0}=g_{0}$ and set $x:=\Gamma_{1} \hat{f}_{0}$. Since $\mathcal{J}\left(\Gamma^{\prime}\right)$ (cf. Section 2.2) is self-adjoint in the Hilbert space $\mathcal{H} \times \mathcal{G}$ there exists $\left\{\hat{f}_{1}, \hat{h}\right\} \in \Gamma^{\prime}$ with $f_{1}^{\prime}-\lambda f_{1}=g_{1}$. Let now $\hat{f}_{\lambda} \in \mathcal{N}_{\lambda, A^{+}}$such that $\Gamma_{0} \hat{f}_{\lambda}=h$ (and hence $\Gamma_{1} \hat{f}_{\lambda}=M(\lambda) h$ ) holds and since $M(\lambda)+\tau(\lambda)$ is surjective by assumption there exists $\hat{l} \in \tau(\lambda)$ with $M(\lambda) l+l^{\prime}=-\left(h^{\prime}+M(\lambda) h+x\right)$. Therefore there are $\hat{f}_{1 \lambda} \in \mathcal{N}_{\lambda, T_{1}}$ and $\hat{f}_{0 \lambda} \in \mathcal{N}_{\lambda, A^{+}}$such that $\left\{\hat{f}_{1 \lambda}, \hat{l}\right\} \in \Gamma^{\prime}$ and $l=\Gamma_{0} \hat{f}_{0 \lambda}$ (and hence $\left.\Gamma_{1} \hat{f}_{0 \lambda}=M(\lambda) l\right)$. Setting

$$
\hat{\mathfrak{f}}_{0}:=\hat{f}_{0}+\hat{f}_{\lambda}+\hat{f}_{0 \lambda} \in A^{+} \text {and } \hat{\mathfrak{f}}_{1}:=\hat{f}_{1}+\hat{f}_{1 \lambda} \in T_{1},
$$

we have $\left\{\hat{\mathfrak{f}}_{1}, \hat{h}+\hat{l}\right\} \in \Gamma^{\prime}$ and from

$$
\left(\begin{array}{c}
\mathfrak{f}_{0}^{\prime}-\lambda \mathfrak{f}_{0} \\
\mathfrak{f}_{1}^{\prime}-\lambda \mathfrak{f}_{1}
\end{array}\right)=\left(\begin{array}{l}
g_{0} \\
g_{1}
\end{array}\right) \text { and }\left(\begin{array}{c}
\Gamma_{0} \hat{\mathfrak{f}}_{0} \\
-\Gamma_{1} \hat{\mathfrak{f}}_{0}
\end{array}\right)=\hat{h}+\hat{l}
$$

we conclude that (3.7) holds, that is, $\widetilde{A}-\lambda$ is surjective.

In Theorem 3.4 below we will impose additional conditions on the symmetric relation $A \in \widetilde{\mathcal{C}}(\mathcal{K})$ and the fixed canonical self-adjoint extension $A_{0}=\operatorname{ker} \Gamma_{0}$ in order to get more information on the (local) spectral properties of the extensions $\widetilde{A}$. For this we briefly recall the notion of locally definitizable self-adjoint relations. 
For a detailed study of (locally) definitizable self-adjoint operators and relations we refer to the papers $[16,19,23]$ of $\mathrm{P}$. Jonas and H. Langer.

If $A_{0}$ is a self-adjoint relation in a Krein space $\mathcal{K}$, then $\lambda \in \mathbb{C}$ belongs to the approximate point spectrum of $A_{0}$, denoted by $\sigma_{a p}\left(A_{0}\right)$, if there exists a sequence $\left(\begin{array}{l}x_{n} \\ y_{n}\end{array}\right) \in A_{0}, n=1,2, \ldots$, such that $\left\|x_{n}\right\|=1$ and $\lim _{n \rightarrow \infty}\left\|y_{n}-\lambda x_{n}\right\|=0$. The extended approximate point spectrum $\widetilde{\sigma}_{a p}\left(A_{0}\right)$ of $A_{0}$ is defined by $\sigma_{a p}\left(A_{0}\right)$ if $0 \notin \sigma_{a p}\left(A_{0}^{-1}\right)$ and by $\sigma_{a p}\left(A_{0}\right) \cup\{\infty\}$ otherwise. A point $\lambda \in \sigma_{a p}\left(A_{0}\right)$ is said to be of positive type (negative type) with respect to $A_{0}$, if for every sequence $\left(\begin{array}{l}x_{n} \\ y_{n}\end{array}\right) \in A_{0}$, $n=1,2 \ldots$, with $\left\|x_{n}\right\|=1, \lim _{n \rightarrow \infty}\left\|y_{n}-\lambda x_{n}\right\|=0$ we have

$$
\liminf _{n \rightarrow \infty}\left[x_{n}, x_{n}\right]_{\mathcal{K}}>0 \quad\left(\limsup _{n \rightarrow \infty}\left[x_{n}, x_{n}\right]_{\mathcal{K}}<0, \text { resp. }\right) \text {. }
$$

If $\infty \in \widetilde{\sigma}_{a p}\left(A_{0}\right), \infty$ is said to be of positive type (negative type) with respect to $A_{0}$ if 0 is of positive type (resp. negative type) with respect to $A_{0}^{-1}$. The set of all spectral points of positive type (negative type) with respect to $A_{0}$ will be denoted by $\sigma_{++}\left(A_{0}\right)$ (resp. $\sigma_{--}\left(A_{0}\right)$ ). An open subset $\Delta$ of $\overline{\mathbb{R}}$ is said to be of positive type (negative type) with respect to $A_{0}$ if $\Delta \cap \widetilde{\sigma}\left(A_{0}\right) \subset \sigma_{++}\left(A_{0}\right)\left(\Delta \cap \tilde{\sigma}\left(A_{0}\right) \subset \sigma_{--}\left(A_{0}\right)\right.$, resp.) holds.

Let in the following $\Omega$ be some domain in $\overline{\mathbb{C}}$ symmetric with respect to the real axis such that $\Omega \cap \overline{\mathbb{R}} \neq \emptyset$ and the intersections of $\Omega$ with the upper and lower open half-planes are simply connected.

Definition 3.3. A self-adjoint relation $A_{0}$ in a Krein space $\mathcal{K}$ is said to be definitizable over $\Omega$ if $\sigma\left(A_{0}\right) \cap(\Omega \backslash \overline{\mathbb{R}})$ consists of isolated points which are poles of the resolvent of $A_{0}$, no point of $\Omega \cap \overline{\mathbb{R}}$ is an accumulation point of the nonreal spectrum of $A_{0}$ in $\Omega$ and the following holds.

(i) For every finite union $\Delta, \bar{\Delta} \subset \Omega \cap \overline{\mathbb{R}}$, of open connected subsets there exists $m \geq 1, M>0$ and an open neighborhood $\mathcal{U}$ of $\bar{\Delta}$ in $\Omega$ such that

$$
\left\|\left(A_{0}-\lambda\right)^{-1}\right\| \leq M(1+|\lambda|)^{2 m-2}|\operatorname{Im} \lambda|^{-m}
$$

holds for all $\lambda \in \mathcal{U} \backslash \overline{\mathbb{R}}$.

(ii) Every point $\mu \in \Omega \cap \overline{\mathbb{R}}$ has an open connected neighborhood $I_{\mu}$ in $\overline{\mathbb{R}}$ such that each component of $I_{\mu} \backslash\{\mu\}$ is either of positive or of negative type with respect to $A_{0}$.

Let $A_{0}$ be definitizable over $\Omega$ and let $e$ be a discrete (possibly empty) set of points in $\Omega \cap \overline{\mathbb{R}}$. Then the property that $(\Omega \cap \overline{\mathbb{R}}) \backslash e$ is of positive type with respect to $A_{0}$ is equivalent to the fact that $\sigma_{--}\left(A_{0}\right)$ is discrete in $\Omega$.

Theorem 3.4. Let $\mathcal{K}$ be a Krein space and let $\mathcal{H}$ be a Hilbert space, let $A \in \widetilde{\mathcal{C}}(\mathcal{K})$ be a symmetric relation of finite defect and let $\left\{\mathcal{G}, \Gamma_{0}, \Gamma_{1}\right\}$ be a boundary triplet for $A^{+}$with corresponding $\gamma$-field $\gamma$ and Weyl function $M$. Assume that $A_{0}=\operatorname{ker} \Gamma_{0}$ is definitizable over $\Omega$ and that $\sigma_{--}\left(A_{0}\right)$ is discrete in $\Omega$.

(i) If $\widetilde{A} \in \widetilde{\mathcal{C}}(\mathcal{K} \times \mathcal{H})$ is a self-adjoint extension of $A$ and $P_{\mathcal{K}}\left(\widetilde{A}-\lambda_{0}\right)^{-1} \uparrow_{\mathcal{K}} \in \mathcal{L}(\mathcal{K})$ holds for some $\lambda_{0} \in \rho\left(A_{0}\right) \cap \Omega$, then there exists a Nevanlinna family $\tau \in \widetilde{\mathcal{R}}(\mathcal{G})$ such that (a)-(c) are satisfied. 
(a) If $\lambda \in \rho\left(A_{0}\right) \cap \Omega$, then $(M(\lambda)+\tau(\lambda))^{-1} \in \mathcal{L}(\mathcal{G})$ if and only if $\lambda \in \rho(\widetilde{A})$.

(b) The formula

$$
P_{\mathcal{K}}(\tilde{A}-\lambda)^{-1}\left\lceil_{\mathcal{K}}=\left(A_{0}-\lambda\right)^{-1}-\gamma(\lambda)(M(\lambda)+\tau(\lambda))^{-1} \gamma(\bar{\lambda})^{+}\right.
$$

holds for all $\lambda \in \rho\left(A_{0}\right) \cap \rho(\widetilde{A}) \cap \Omega$.

(c) If $\rho(\widetilde{A}) \cap \Omega \neq \emptyset$ then $\widetilde{A}$ is definitizable over $\Omega$ and $\sigma_{--}(\widetilde{A})$ is discrete in $\Omega$.

(ii) If $\tau \in \widetilde{\mathcal{R}}(\mathcal{G})$ is a Nevanlinna family and $\left(M\left(\lambda_{0}\right)+\tau\left(\lambda_{0}\right)\right)^{-1} \in \mathcal{L}(\mathcal{G})$ for some $\lambda_{0} \in \rho\left(A_{0}\right) \cap \Omega$, then there exists a self-adjoint extension $\widetilde{A} \in \widetilde{\mathcal{C}}(\mathcal{K} \times \mathcal{H})$ of $A$ such that (a)-(c) are satisfied.

Proof. The statement of Theorem 3.4 follows from Theorem 3.2 if we show that the extension $\widetilde{A}$ in (i) satisfies assertion (c).

For this, let $S_{0}$ and $S_{1}$ be the symmetric relations in the Krein space $\mathcal{K}$ and the Hilbert space $\mathcal{H}$, respectively, defined in step 1 of the proof of Theorem 3.1. As $A$ is of finite defect the deficiency indices $n_{ \pm}\left(J_{\mathcal{K}} A\right)$ of the symmetric relation $J_{\mathcal{K}} A$ in the Hilbert space $\left(\mathcal{K},\left[J_{\mathcal{K}} \cdot, \cdot\right]\right)$ are both equal to $n<\infty$ and hence the deficiency indices $n_{ \pm}\left(J_{\mathcal{K}} S_{0}\right)$ of the symmetric relation $J_{\mathcal{K}} S_{0}$ are both equal to $m \leq n$. Considerations very similar to those in [9, Lemma 2.14] show that the deficiency indices $n_{ \pm}\left(S_{1}\right)$ of $S_{1}$ coincide and are also equal to $m$.

Let $B_{0}$ be a self-adjoint extension of $S_{1}$ in the Hilbert space $\mathcal{H}$. We claim that the self-adjoint relation $A_{0} \times B_{0} \in \widetilde{\mathcal{C}}(\mathcal{K} \times \mathcal{H})$ is definitizable over $\Omega$ and $\sigma_{--}\left(A_{0} \times B_{0}\right)$ is discrete in $\Omega$. In fact, first of all $\sigma\left(A_{0} \times B_{0}\right) \cap(\Omega \backslash \overline{\mathbb{R}})$ coincides with $\sigma\left(A_{0}\right) \cap(\Omega \backslash \overline{\mathbb{R}})$ and the growth properties of the resolvent of $A_{0}$ and $B_{0}$, $\left\|\left(B_{0}-\lambda\right)^{-1}\right\| \leq|\operatorname{Im} \lambda|^{-1}, \lambda \notin \mathbb{R}$, imply that condition (i) in Definition 3.3 holds for $A_{0} \times B_{0}$. Moreover $\overline{\mathbb{R}} \subset \sigma_{++}\left(B_{0}\right) \cup \widetilde{\rho}\left(B_{0}\right)$ and the assumptions that $A_{0}$ is definitizable over $\Omega$ and $\sigma_{--}\left(A_{0}\right)$ is discrete in $\Omega$ imply that with the exception of a discrete set $\Omega \cap \overline{\mathbb{R}}$ belongs to $\sigma_{++}\left(A_{0} \times B_{0}\right) \cup \widetilde{\rho}\left(A_{0} \times B_{0}\right)$. Therefore $A_{0} \times B_{0}$ is definitizable over $\Omega$ and $\sigma_{--}\left(A_{0} \times B_{0}\right)$ is discrete in $\Omega$.

Since $\widetilde{A}$ and $A_{0} \times B_{0}$ are self-adjoint extensions of the symmetric relation $S_{0} \times S_{1}$ in $\mathcal{K} \times \mathcal{H}$ and $\rho(\widetilde{A}) \cap \Omega$ is nonempty we conclude that

$$
(\widetilde{A}-\lambda)^{-1}-\left(\left(A_{0} \times B_{0}\right)-\lambda\right)^{-1}, \quad \lambda \in \rho(\widetilde{A}) \cap \rho\left(A_{0} \times B_{0}\right) \cap \Omega,
$$

is a finite rank operator. Hence we can apply [2, Theorem 2.2] and it follows that $\widetilde{A}$ is definitizable over $\Omega$ and $\sigma_{--}(\widetilde{A})$ is discrete in $\Omega$.

\section{Realization of relation-valued functions as Weyl families}

We show in Theorem 4.1 that certain classes of $\widetilde{\mathcal{C}}(\mathcal{G})$-valued functions can be realized as Weyl families corresponding to boundary relations $\Gamma \in \widetilde{\mathcal{C}}\left(\mathcal{K}^{2}, \mathcal{G}^{2}\right)$ of symmetric relations in Krein spaces $\mathcal{K}$. Lemma 2.4 (i) and the proof of [9, Theorem 3.9] suggest that for a given $\widetilde{\mathcal{C}}(\mathcal{G})$-valued function $\tau$ the function $\lambda \mapsto-(\tau(\lambda)+\lambda)^{-1}$ 
has to be realized as the compressed resolvent of some self-adjoint relation $\mathcal{J}(\Gamma)$ in $\mathcal{K} \times \mathcal{G}$.

We briefly recall the notion of (locally) definitizable functions introduced and studied by $\mathrm{P}$. Jonas in $[17,18,19,20]$. Let, as in Definition $3.3, \Omega$ be a domain which is symmetric with respect to the real axis such that $\Omega \cap \overline{\mathbb{R}} \neq \emptyset$ and the intersections of $\Omega$ with the upper and lower open half-planes are simply connected. For an $\mathcal{L}(\mathcal{G})$ valued function $G$ meromorphic in $\Omega \backslash \overline{\mathbb{R}}$ we denote the set of all $\lambda \in \Omega \backslash\{\infty\}$ such that $\tau$ can analytically be continued in $\lambda$ in a unique way by $\mathfrak{h}(G)$.

An $\mathcal{L}(\mathcal{G})$-valued function $G$ meromorphic in $\mathbb{C} \backslash \mathbb{R}$ satisfying $G(\bar{\lambda})=G(\lambda)^{*}$ for all $\lambda \in \mathbb{C} \backslash \mathbb{R}$ is called definitizable if there exists a scalar rational function $r$ such that $r G$ is the sum of a Nevanlinna function $N$ and an $\mathcal{L}(\mathcal{G})$-valued rational function $n$ whose poles belong to $\mathfrak{h}(G)$,

$$
r(\lambda) G(\lambda)=N(\lambda)+n(\lambda)
$$

for all points $\lambda \in \mathbb{C} \backslash \mathbb{R}$ of holomorphy of $r G$, cf. [18, $\S 3]$. If $\Omega$ is a domain as above, then an $\mathcal{L}(\mathcal{G})$-valued function $G$ meromorphic in $\Omega \backslash \overline{\mathbb{R}}$ satisfying $G(\bar{\lambda})=G(\lambda)$ * for all $\lambda \in \Omega \backslash \overline{\mathbb{R}}$ is said to be locally definitizable in $\Omega$, if for every domain $\Omega^{\prime}$ with the same properties as $\Omega, \overline{\Omega^{\prime}} \subset \Omega, G$ can be written as the sum $G_{d}+G_{h}$ of a definitizable function $G_{d}$ and a function $G_{h}$ locally holomorphic on $\Omega^{\prime}$ (see [20]).

Theorem 4.1. Let $\mathcal{G}$ be a Hilbert space, let $\tau$ be a $\widetilde{\mathcal{C}}(\mathcal{G})$-valued family and assume that the function

$$
\lambda \mapsto G(\lambda):=-(\tau(\lambda)+\lambda)^{-1}
$$

is an $\mathcal{L}(\mathcal{G})$-valued locally definitizable function in $\Omega$. Then for every domain $\Omega^{\prime}$ with the same properties as $\Omega, \overline{\Omega^{\prime}} \subset \Omega$, there exists a Krein space $\mathcal{K}$, a closed symmetric relation $A \in \widetilde{\mathcal{C}}(\mathcal{K})$ and a boundary relation $\Gamma \in \widetilde{\mathcal{C}}\left(\mathcal{K}^{2}, \mathcal{G}^{2}\right)$ for $A^{+}$such that the corresponding Weyl family coincides with $\tau$ in $\Omega^{\prime} \cap \mathfrak{h}(G)$.

Proof. Let us fix some domain $\Omega^{\prime}, \overline{\Omega^{\prime}} \subset \Omega$, and a point $\lambda_{0} \in \Omega^{\prime} \cap \mathfrak{h}(G)$. Since $G$ is a definitizable function in $\Omega$ by [1] the same holds for the function

$$
G_{1}(\lambda):=\lambda-\operatorname{Re} \lambda_{0}+\left(\lambda-\lambda_{0}\right)\left(\lambda-\bar{\lambda}_{0}\right) G(\lambda), \quad \lambda \in \Omega \cap \mathfrak{h}(G) .
$$

Hence [20, Theorem 3.8] implies that there exists a Krein space $\widetilde{\mathcal{K}}$, a self-adjoint relation $B \in \widetilde{\mathcal{C}}(\widetilde{\mathcal{K}})$ definitizable over $\Omega$, and a mapping $\gamma \in \mathcal{L}(\mathcal{G}, \widetilde{\mathcal{K}})$ such that $\Omega^{\prime} \cap \mathfrak{h}\left(G_{1}\right)=\Omega^{\prime} \cap \rho(B)$ and

$$
G_{1}(\lambda)=\operatorname{Re} G_{1}\left(\lambda_{0}\right)+\gamma^{+}\left(\lambda-\operatorname{Re} \lambda_{0}+\left(\lambda-\lambda_{0}\right)\left(\lambda-\bar{\lambda}_{0}\right)(B-\lambda)^{-1}\right) \gamma
$$

holds for all $\lambda \in \Omega^{\prime} \cap \mathfrak{h}\left(G_{1}\right)$. By (4.1) we have $G_{1}\left(\lambda_{0}\right)=i \operatorname{Im} \lambda_{0}$ and $\operatorname{Re} G_{1}\left(\lambda_{0}\right)=0$ which together with (4.2) yields $\gamma^{+} \gamma=I_{\mathcal{G}}$. Since $\gamma \gamma^{+}$is a self-adjoint projection in the Krein space $\widetilde{\mathcal{K}}$ we can identify $\mathcal{G}$ with the Hilbert subspace ran $\gamma$ in $\widetilde{\mathcal{K}}$. Then the orthogonal companion $\mathcal{K}:=\mathcal{G}^{[\perp]}$ of $\mathcal{G}$ in $\widetilde{\mathcal{K}}$ is a Krein space and $\widetilde{\mathcal{K}}=\mathcal{K}[\dot{+}] \mathcal{G}$ holds. Moreover $\gamma$ is the embedding of $\mathcal{G}$ into $\widetilde{\mathcal{K}}$ and $\gamma^{+}$the projection $P_{\mathcal{G}}$ in $\widetilde{\mathcal{K}}$ onto $\mathcal{G}$. Hence $(4.2)$ can be rewritten as

$$
G_{1}(\lambda)=\lambda-\operatorname{Re} \lambda_{0}+\left(\lambda-\lambda_{0}\right)\left(\lambda-\bar{\lambda}_{0}\right) P_{\mathcal{G}}(B-\lambda)^{-1} \Upsilon_{\mathcal{G}}
$$


and taking into account (4.1) we conclude

$$
-(\tau(\lambda)+\lambda)^{-1}=G(\lambda)=P_{\mathcal{G}}(B-\lambda)^{-1} \uparrow_{\mathcal{G}}, \quad \lambda \in \Omega^{\prime} \cap \mathfrak{h}(G) .
$$

Let $\mathcal{J}$ be as in $(2.3)$ and define $\Gamma:=\mathcal{J}^{-1}(B)$. Then $\Gamma$ is a boundary relation for $A^{+}, A:=\operatorname{ker} \Gamma$, and by (2.4) the associated Weyl family is $\tau$.

Remark 4.2. The self-adjoint relation $B=\mathcal{J}(\Gamma)$ corresponding to the boundary relation $\Gamma$ (with Weyl family $\tau$ ) constructed in the proof of Theorem 4.1 is definitizable over $\Omega^{\prime}$. A converse statement also holds, that is, if $\Gamma$ is a boundary relation such that $\mathcal{J}(\Gamma)$ is definitizable over $\Omega$, then the corresponding Weyl family meets the assumptions of Theorem 4.1 .

By virtue of [1, Theorem 2.5] we immediately obtain the following corollary on matrix-valued locally definitizable functions.

Corollary 4.3. Let $\tau$ be a matrix-valued definitizable function in $\Omega$ and assume that $\operatorname{det}(\tau(\lambda)+\lambda)$ is not identically equal to zero. Then for every domain $\Omega^{\prime}$ as $\Omega, \overline{\Omega^{\prime}} \subset \Omega$, there exists a Krein space $\mathcal{K}$, a closed symmetric relation $A$ in $\mathcal{K}$ and boundary relation $\Gamma \in \widetilde{\mathcal{C}}\left(\mathcal{K}^{2}, \mathcal{G}^{2}\right)$ such that the corresponding Weyl family coincides with $\tau$ in $\Omega^{\prime}$.

A similar construction as in the proof of Theorem 4.1 yields an alternative proof of the main realization theorem in [9].

Corollary 4.4. Every Nevanlinna family $\tau \in \widetilde{\mathcal{R}}(\mathcal{G})$ can be realized as the Weyl family of a boundary relation $\Gamma \in \widetilde{\mathcal{C}}\left(\mathcal{K}^{2}, \mathcal{G}^{2}\right)$, where $\mathcal{K}$ is a Hilbert space.

Proof. As the sum and the negative inverse of a Nevanlinna family are Nevanlinna families

$$
\begin{aligned}
G_{1}(\lambda): & =\lambda-\left(\lambda^{2}+1\right)(\tau(\lambda)+\lambda)^{-1} \\
& =-\left\{-\left(\lambda-\tau(\lambda)^{-1}\right)^{-1}-\left(\tau(\lambda)-\lambda^{-1}\right)^{-1}\right\}^{-1},
\end{aligned}
$$

$\lambda \in \mathbb{C} \backslash \mathbb{R}$, is an $\mathcal{L}(\mathcal{G})$-valued Nevanlinna function. Hence there exists a Hilbert space $\widetilde{\mathcal{K}}$, a self-adjoint relation $B \in \widetilde{\mathcal{C}}(\widetilde{\mathcal{K}})$ and an operator $\gamma \in \mathcal{L}(\mathcal{G}, \widetilde{\mathcal{K}})$ such that a representation of the form (4.2) with $\lambda_{0}=i$ holds. Now the same reasoning as in the proof of Theorem 4.1 shows that $\Gamma=\mathcal{J}^{-1}(B)$ is a boundary relation with Weyl family $\tau$.

\section{Acknowledgement}

The authors thank P. Jonas for fruitful discussions and valuable remarks.

\section{References}

[1] T.Ya. Azizov, P. Jonas: On locally definitizable matrix functions, Preprint 21 (2005), Preprint Series TU Berlin.

[2] J. Behrndt: Finite rank perturbations of locally definitizable self-adjoint operators in Krein spaces, to appear in J. Operator Theory. 
[3] J. Behrndt, A. Luger, C. Trunk: Generalized resolvents of a class of symmetric operators in Krein spaces, to appear in Operator Theory: Advances and Applications.

[4] J. Behrndt, C. Trunk: On generalized resolvents of symmetric operators of defect one with finitely many negative squares, Proceedings AIT Conference, University of Vaasa, Finland, 124 (2005), 21-30.

[5] V.A. Derkach: On Weyl function and generalized resolvents of a hermitian operator in a Krein space, Integral Equations Operator Theory 23 (1995), 387-415.

[6] V.A. Derkach: On Krein space symmetric linear relations with gaps, Methods Funct. Anal. Topology 4 (1998), 16-40.

[7] V.A. Derkach: On generalized resolvents of hermitian relations in Krein spaces, J. Math. Sci. (New York) 97 (1999), 4420-4460.

[8] V.A. Derkach, S. Hassi, M.M. Malamud, H.S.V. de Snoo: Generalized resolvents of symmetric operators and admissibility, Methods Funct. Anal. Topology 6 (2000), $24-53$.

[9] V.A. Derkach, S. Hassi, M.M. Malamud, H.S.V. de Snoo: Boundary relations and their Weyl families, Trans. Amer. Math. Soc. 358 (2006), 5351-5400.

[10] V.A. Derkach, S. Hassi, M.M. Malamud, H.S.V. de Snoo: Boundary relations and orthogonal coupling of symmetric operators, Proceedings AIT Conference, University of Vaasa, Finland, 124 (2005), 41-56.

[11] V.A. Derkach, S. Hassi, M.M. Malamud, H.S.V. de Snoo: Boundary relations and generalized resolvents of symmetric operators, preprint.

[12] V.A. Derkach, M.M. Malamud: Generalized resolvents and the boundary value problems for hermitian operators with gaps, J. Funct. Anal. 95 (1991), 1-95.

[13] V.A. Derkach, M.M. Malamud: The extension theory of hermitian operators and the moment problem, J. Math. Sci. (New York) 73 (1995), 141-242.

[14] A. Dijksma, H.S.V. de Snoo: Symmetric and self-adjoint relations in Krein spaces I, Operator Theory: Advances and Applications 24, Birkhäuser Verlag Basel (1987), 145-166.

[15] V.I. Gorbachuk, M.L. Gorbachuk: Boundary Value Problems for Operator Differential Equations, Kluwer Academic Publishers, Dordrecht (1991).

[16] P. Jonas: On a class of unitary operators in Krein space. Advances in Invariant Subspaces and Other Results of Operator Theory, Operator Theory: Advances and Applications 17, Birkhäuser Verlag Basel (1986), 151-172.

[17] P. Jonas: A class of operator-valued meromorphic functions on the unit disc, Ann. Acad. Scie. Fenn. Math. 17 (1992), 257-284.

[18] P. Jonas: Operator representations of definitizable functions, Ann. Acad. Scie. Fenn. Math. 25 (2000), 41-72.

[19] P. Jonas: On locally definite operators in Krein spaces, in: Spectral Theory and Applications, Theta Foundation (2003), 95-127.

[20] P. Jonas: On operator representations of locally definitizable functions, Operator Theory: Advances and Applications 162, Birkhäuser Verlag Basel (2005), 165-190.

[21] M.G. Krein, H. Langer: On defect subspaces and generalized resolvents of Hermitian operators in Pontryagin spaces, Funktsional. Anal. i Prilozhen. 5 No. 2 (1971) 59-71; 
5 No. 3 (1971) 54-69 (Russian); English transl.: Funct. Anal. Appl. 5 (1971/1972), 139-146, 217-228.

[22] H. Langer: Verallgemeinerte Resolventen eines $J$-nichtnegativen Operators mit endlichem Defekt, J. Funct. Anal. 8 (1971), 287-320.

[23] H. Langer: Spectral functions of definitizable operators in Krein spaces, Functional Analysis Proceedings of a Conference held at Dubrovnik, Yugoslavia, November 214, 1981, Lecture Notes in Mathematics 948, Springer Verlag Berlin-Heidelberg-New York (1982), 1-46.

[24] H. Langer, B. Textorius: On generalized resolvents and $Q$-functions of symmetric linear relations (subspaces) in Hilbert space, Pacific J. Math. 72 (1977), 135-165.

[25] M.G. Krein: On hermitian operators with defect-indices equal to unity, Dokl. Akad. Nauk SSSR, 43 (1944), 339-342.

[26] M.G. Krein: On the resolvents of an hermitian operator with defect-index $(m, m)$, Dokl. Akad. Nauk SSSR, 52 (1946), 657-660.

[27] M.M. Malamud: On a formula for the generalized resolvents of a non-densely defined Hermitian Operator, Ukrain. Mat. Zh. 44 (1992), 1658-1688 (Russian); English transl.: Ukrainian Math. J. 44 (1993), 1522-1547.

[28] M.A. Naimark: On spectral functions of a symmetric operator, Izv. Akad. Nauk SSSR, Ser. Matem. 7 (1943), 373-375.

Jussi Behrndt

Institut für Mathematik, TU Berlin, Straße des 17. Juni 136, 10623 Berlin, Germany

e-mail: behrndt@math.tu-berlin.de

Hans-Christian Kreusler

Institut für Mathematik, TU Berlin, Straße des 17. Juni 136, 10623 Berlin, Germany

e-mail: kreusler@math.tu-berlin.de 